\title{
Mitofusins: ubiquitylation promotes fusion
}

\author{
Cell Research (2014) 24:387-388. doi:10.1038/cr.2014.23; published online 21 Februay 2014
}

Mitochondrial genes including $M f n 2$ are at the center of many diseases, underscoring their potential as a therapeutical target. The Chen group now identified 15-oxospiramilactone as a chemical inhibitor of the mammalian deubiquitylase USP30, acting on Mfn1 and Mfn2.

Mitofusins, Fzo1 in yeast and Mfn1 and Mfn2 in mammals, are ubiquitylated and this post-translational modification has both positive and negative consequences on mitochondrial fusion [1]. The process of ubiquitylation requires enzymes belonging to three classes of proteins called E1, E2 and E3, which catalyze a cascade of successive steps leading to the covalent attachment of the modifier to its target protein [2]. Deubiquitylating enzymes render this modification reversible, thus offering further possibilities for regulation [2]. Ubiquitylation of mitofusins leads to their proteolyic breakdown, inhibiting fusion of mitochondria that consequently undergo fragmentation (Figure 1, left panel) [1,3]. For example in response to mitochondrial depolarization or apoptotic stimuli, E3 ligases like Parkin and Huwe1 ubiquitylate and target Mfn1 and Mfn2 to the proteasome (Figure 1, left panel) [3, 4]. However, ubiquitylation of mitofusins is a dual process and a non-proteolytic role of mitofusin ubiquitylation that promotes mitochondrial fusion is now emerging [1]. This opposing mechanism was first described in yeast, where the isopeptidases Ubp12 and Ubp2 that deubiquitylate Fzo1 have been identified [5]. Inhibition and activation of mitochondrial fusion by ubiquitylation enable different morphologies of mitochondria ranging from a multitude of small organelles to a hyperconnected network (Figure 1) [5]. In a recent paper published in Cell Research, Yue et al. [6] reveal that a similar process is present in mammalian cells. The authors report that the isopeptidase USP30 acts on ubiquitylated forms of Mfn1 and Mfn2 that stimulate mitochondrial fusion (Figure 1, right panel). This discovery identifies for the first time in mammals a positive role of ubiquitylation in the regulation of Mfn1 and Mfn2 fusion activity [6].

Moreover, Yue et al. [6] identified the first small molecule inhibitor of mitochondrial fusion, 15-oxospiramilactone, which targets USP30 in both human and mouse cell lines. 15-oxospiramilactone is a semi-synthetic diterpene alkaloid of $330 \mathrm{Da}$ that can be chemically synthetized through an oxidation reaction from spiramines extracted from the roots of a Chinese herbal medicine Spiraea japonica (Rosaceae). Inhibition of USP30 increased ubiquitylation of Mfn1 and Mfn2 and led to an elongation of the mitochondrial network (Figure 1, right panel) $[6,7]$. USP30 is a cysteine ubiquitin isopeptidase $\mathrm{N}$-terminally anchored to the outer membrane of mitochondria, which was previously shown to regulate mitochondrial morphology dependent on Mfn1 and Mfn2 [7]. USP30 knockdown leads to mitochondrial elongation, a phenotype rescued by ectopic expression of wild-type USP30, while the catalytically inactive mutant C77S USP30 failed to revert [7]. Yue et al. [6] show that 15-oxospiramilactone directly interacts with USP30, which also depends on its catalytically active cysteine, and inhibits the DUB activity of USP30 on tetraubiquitin chains. Moreover, they demonstrate that inhibition of USP30 and subsequent mitochondrial elongation are due to stimulated mitochondrial fusion activity, apparently with no influence on mitochondrial fission [6]. Concomitantly, cells showed increased ubiquitylation of Mfn1 and Mfn2 without significant changes in protein turnover of these two proteins [6]. Therefore, in analogy to findings in yeast, ubiquitylation of Mfn1 and Mfn2 can either signal them to activate mitochondrial fusion or in contrast promote their proteasomal degradation, resulting in mitochondrial fission (Figure 1).

Importantly, 15-oxospiramilactone reverts the mitochondrial fragmentation phenotype of single Mfn-knockout ( $M f n 1^{-/}$or $M f n 2^{-/}$) cells, suggesting that mitochondrial fusion depends on the ubiquitylation of both mitofusin proteins [6]. In yeast, the importance of ubiquitylation was proven by directly attaching a deubiquitylase to Fzo1, which resulted in a non-ubiquitylated and non-functional Fzo1 protein [5]. In addition, the identification and the subsequent mutagenesis study of the ubiquitylation sites in Fzol confirmed an interplay between ubiquitylation and oligomerization in mitochondrial fusion in S. cerevisiae [5]. Impairing the yeast $\mathrm{E} 3$ ligase $\mathrm{SCF}^{\mathrm{Mdm} 30}$ inhibited mitochondrial fusion and, conversely, ablation of $U B P 12$ led to more fusion events $[5,8]$. Given this new identification of USP30 as the functional orthologue of the yeast Ubp12, future studies will certainly aim at the identification of the E3 ligase counterpart of $\mathrm{SCF}^{\mathrm{Mdm} 30}$ and ubiquitylation sites in Mfn1 and Mfn2. In addition to USP30 inhibition, other 


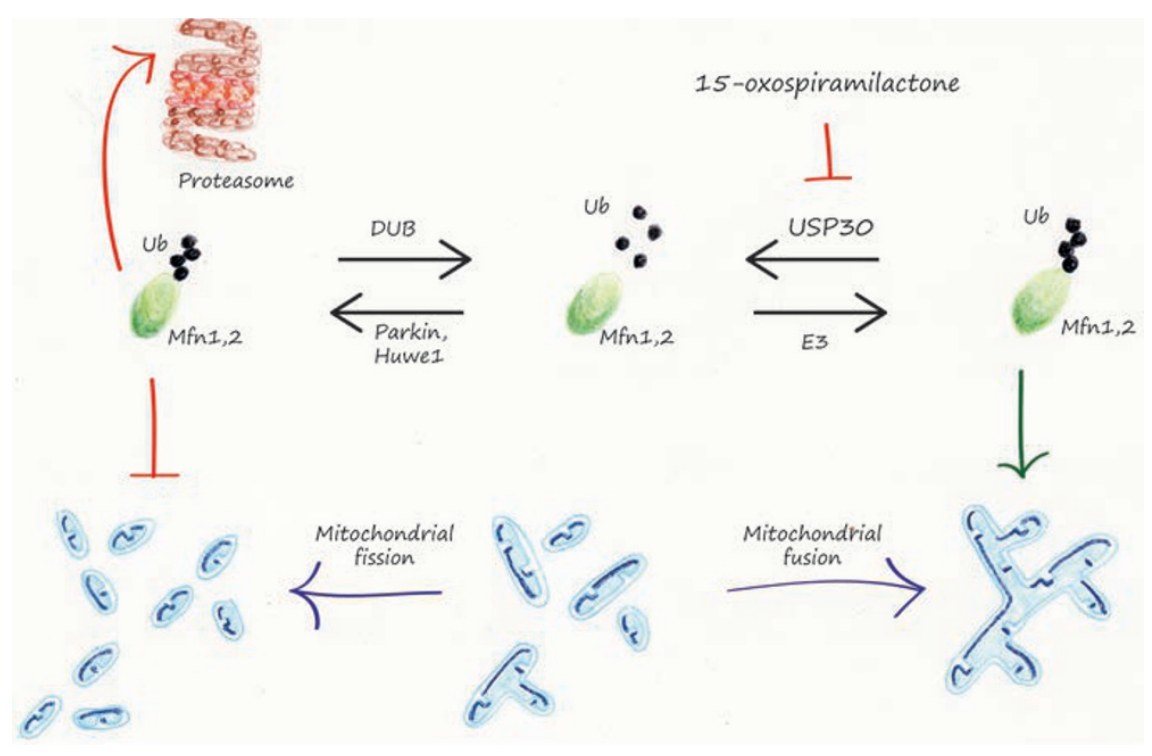

Figure 1 Dual roles of ubiquitylation and deubiquitylation of mitofusins Mfn1 and Mfn2, the key effectors for mitochondrial fusion, in regulating mitochondrial fusion. On one hand, ubiquitylation of Mfn1 and Mfn2 by E3 ligases like Parkin or Huwe1 targets their proteasomal degradation and inhibits mitochondrial fusion, which results in mitochondrial fragmentation due to unopposed fission events. On the other hand, ubiquitylation of Mfn1 and Mfn2 by an unknown E3 ligase enhances their activity and promotes mitochondrial fusion. This positive regulation is counteracted by the deubiquitylase USP30, targeted by the small molecule inhibitor 15-oxospiramilactone.

conditions leading to mitochondrial hyperfusion have been previously observed, such as mild stress conditions that increase reactive oxygen species (ROS) [9]. Importantly, oxidative stress and mitochondrial fusion are directly linked as ROS induces disulphide switching of Mfn2 to oligomeric forms that promote mitochondrial fusion [9]. It would be interesting to investigate whether 15-oxospiramilactone also affects the generation of disulphidemediated mitofusin oligomers, thus activating mitochondrial fusion.

Mutations in Mfn2 are causative for the Charcot-Marie-Tooth type 2A neuropathy, an autosomal dominant disorder of the peripheral nervous system that mainly affects axons and lower ex- tremities [1]. Deficiencies in Parkin and Mfn2 ubiquitylation were also linked to Parkinson's disease [3]. In addition to neuropathies, Mfn2 is associated to other diseases like cardiomyophathies and diabetes [1]. Yue et al. [6] found that 15-oxospiramilactone reverted phenotypes arising from the lack of Mfn1 or Mfn2. It restored the normal distribution of mtDNA, allowed recovery of the $\Delta \Psi \mathrm{m}$ and increased the ATP levels and OXPHOS capacity of the rebuilt mitochondrial network. Therefore, this study potentiates 15-oxospiramilactone for therapeutical benefit. The anti-cancer properties of 15-oxospiramilactone, also named S3 or NC043, have been previously reported $[10,11]$. It inhibits $\mathrm{Wnt} / \beta$-catenin signaling and colon cancer cell tumorigenesis in a xenograft model [10]. Moreover, 15-oxospiramilactone increases Bim expression and apoptosis to inhibit tumor growth from $\mathrm{Bax}^{-/-} / \mathrm{Bak}^{-/-}$cells implanted in mice [11]. However, Yue et al. [6] show that the effect of 15-oxospiramilactone in mitochondrial fusion is independent of apoptosis and suggest that the difference is due to drug concentration. Indeed, previous anti-cancer studies used 15-oxospiramilactone at a concentration range of 3.75-15 $\mu \mathrm{M}[10,11]$, whereas 2 $\mu \mathrm{M}$ suffice to inhibit USP30 [6]. Further studies are needed to address the clinical relevance of 15-oxospiramilactone and USP30 in Mfn2-associated diseases.

\section{Mafalda Escobar-Henriques ${ }^{1}$ \\ ${ }^{\prime}$ Institute for Genetics, Centre for Molecular Medicine (CMMC), Cologne Excellence Cluster on Cellular Stress Responses in Aging-Associat- ed Diseases (CECAD), University of Cologne. Zülpicher Str 47a, 50674 Cologne, Germany Correspondence: Mafalda Escobar-Henriques E-mail: Mafalda.Escobar@uni-koeln.de}

\section{References}

1 Escobar-Henriques M, Anton F. Biochim Biophys Acta 2013; 1883:162-175.

2 Komander D, Rape M. Annu Rev Biochem 2012; 81:203-229.

3 Tanaka A, Cleland MM, Xu S, et al. J Cell Biol 2010; 191:1367-1380.

4 Leboucher GP, Tsai YC, Yang M, et al. Mol Cell 2012; 47:547-557.

5 Anton F, Dittmar G, Langer T, et al. Mol Cell 2013; 49:487-498.

6 Yue W, Chen G, Liu H, et al. Cell Res 2014; 24:482-496.

7 Nakamura N, Hirose S. Mol Biol Cell 2008; 19:1903-1911.

8 Cohen MM, Leboucher GP, Livnat-Levanon N, et al. Mol Biol Cell 2008; 19:2457-2464.

9 Shutt T, Geoffrion M, Milne R, et al. EMBO Rep 2012; 13: 909-915.

10 Wang W, Liu H, Wang S, et al. Cell Res 2011; 21:730-740.

11 Zhao L, He F, Liu H, et al. J Biol Chem 2012; 287:1054-1065. 